\title{
A new class of q-Fibonacci polynomials
}

\author{
Johann Cigler
}

\author{
Institut für Mathematik \\ Universität .Wien \\ A - 1090 Wien, Osterreich \\ Johann.Cigler@univie.ac.at
}

Submitted: Mar 24, 2003; Accepted: May 2, 2003; Published: May 7, 2003

MR Subject Classifications: primary 05A30, 05A15; secondary: 15A15

\begin{abstract}
We introduce a new $q$-analogue of the Fibonacci polynomials and derive some of its properties. Extra attention is paid to a special case which has some interesting connections with Euler's pentagonal number theorem.
\end{abstract}

\section{Introduction}

The Fibonacci polynomials $f_{n}(x, s)$ are defined by the recursion $f_{n}(x, s)=x f_{n-1}(x, s)+$ $s f_{n-2}(x, s)$ with initial values $f_{0}(x, s)=0, f_{1}(x, s)=1$. They are given by the explicit formula $f_{n}(x, s)=\sum_{k=0}^{\left\lfloor\frac{n-1}{2}\right\rfloor}\left(\begin{array}{c}n-k-1 \\ k\end{array}\right) x^{n-1-2 k} s^{k}$. L. Carlitz [3] has defined a $q$-analogue, which has been extensively studied (cf. e.g. [6], [2], [8]).

In [7] I found that $F_{n}(x, s)=\sum_{k=0}^{\left\lfloor\frac{n-1}{2}\right\rfloor}\left[\begin{array}{c}n-k-1 \\ k\end{array}\right] q^{\left(\begin{array}{c}(k+1 \\ 2\end{array}\right)} x^{n-1-2 k} s^{k}$ is another natural $q$ analogue which satisfies the simple but rather unusual recursion (2.8). This recursion does not lend itself to the computation of special values. Therefore I was surprised as I learned that it has been shown in [9] and [13] that $F_{n}\left(1,-\frac{1}{q}\right)=\sum_{k=0}^{\left\lfloor\frac{n-1}{2}\right\rfloor}(-1)^{k} q^{\left(\begin{array}{c}k \\ 2\end{array}\right)}\left[\begin{array}{c}n-1-k \\ k\end{array}\right]$ has the simple evaluation (3.2). This fact led me to a thorough study of this q-analogue via a combinatorial approach based on Morse code sequences. We show that these q-Fibonacci polynomials satisfy some other recurrences too, generalize some well-known facts for ordinary Fibonacci polynomials to this case, derive their generating function and study the special values $F_{n}\left(1,-\frac{1}{q}\right)$ and $F_{n}(1,-1)$ which turn out to be intimately connected with Euler's pentagonal number series. Finally we show that the Hankel determinants $\operatorname{det}\left(F_{i+j+k}\left(1,-\frac{1}{q}\right)\right)_{i, j=0}^{n-1}$ can be explicitly evaluated. 
I want to thank H. Prodinger for pointing out to me identity (4.7) in [1] and the paper [4], S.O. Warnaar for some helpful remarks and drawing my attention to [9], and R. Chapman and C. Krattenthaler for providing another simple proof of (3.2).

Morse code sequences are finite sequences of dots $(\bullet)$ and dashes $(-)$. We assume that a dot has length 1 and a dash has length 2. The number of all such sequences of total length $n-1$ is the Fibonacci number $F_{n}$, which is defined as the sequence of numbers satisfying the recursion $F_{n}=F_{n-1}+F_{n-2}$ with initial conditions $F_{0}=0$ and $F_{1}=1$.

Let $M C$ be the set of all Morse code sequences. We interpret $M C$ as a monoid with respect to concatenation whose unit element is the empty sequence $\varepsilon$. If we write $a$ for a dot and $b$ for a dash then $M C$ consists of all words in $a$ and $b$. Let $P$ be the corresponding monoid algebra over $\mathbb{C}$, i.e. the algebra of all finite sums $\sum_{v \in M C} \lambda_{v} v$ with complex coefficients.

An important element of $P$ is the binomial

$$
(a+b)^{n}=\sum_{k=0}^{n} C_{k}^{n}(a, b)
$$

Here $C_{k}^{n}(a, b)$ is the sum of all words with $k$ dashes and $n-k$ dots. It is characterized by the boundary values $C_{k}^{0}(a, b)=\delta_{k, 0}$ and $C_{0}^{n}(a, b)=a^{n}$ and each of the two recursions

$$
C_{k}^{n+1}(a, b)=b C_{k-1}^{n}(a, b)+a C_{k}^{n}(a, b)
$$

or

$$
C_{k}^{n+1}(a, b)=C_{k-1}^{n}(a, b) b+C_{k}^{n}(a, b) a .
$$

It is clear that the image of $C_{k}^{n}(a, b)$ under the homomorphism $\varphi: P \rightarrow \mathbb{C}$, defined by $\varphi(a)=\varphi(b)=1$, is the binomial coefficient $\left(\begin{array}{l}n \\ k\end{array}\right)$.

Let $R$ be the ring of linear operators on the vector space of polynomials $\mathbb{C}[x, s]$. We are interested in multiplication operators with polynomials and the operator $\varepsilon$ in $R$ defined by $\varepsilon f(s)=f(q s)$. Let now

$$
\varphi(a)=x \varepsilon, \varphi(b)=q s \varepsilon
$$

Then $\varphi(a) \varphi(b)=q \varphi(b) \varphi(a)$.

The q-binomial theorem (see e.g.[5]) states that for $n \in \mathbb{N}$ $(A+B)^{n}=\sum\left[\begin{array}{l}n \\ k\end{array}\right] B^{k} A^{n-k}$ if $A B=q B A$.

Here $\left[\begin{array}{l}n \\ k\end{array}\right]=\frac{\left(q^{n}-1\right) \cdots\left(q^{n-k+1}-1\right)}{\left(q^{k}-1\right) \cdots(q-1)}$ denotes the q-binomial coefficient or Gaussian polynomial (cf. e.g. [1] or [5]).

Therefore we get the well-known result

$$
(x+q s)\left(x+q^{2} s\right) \cdots\left(x+q^{n} s\right) \varepsilon^{n}=(x \varepsilon+q s \varepsilon)^{n}=\sum_{k=0}^{n}\left[\begin{array}{l}
n \\
k
\end{array}\right] q^{\left(\begin{array}{c}
k+1 \\
2
\end{array}\right)} s^{k} x^{n-k} \varepsilon^{n}
$$

and as special case 


$$
\varphi\left(C_{k}^{n}(a, b)\right)=\left[\begin{array}{l}
n \\
k
\end{array}\right](q s \varepsilon)^{k}(x \varepsilon)^{n-k}=\left[\begin{array}{l}
n \\
k
\end{array}\right] q^{\left(\begin{array}{c}
k+1 \\
2
\end{array}\right)} s^{k} x^{n-k} \varepsilon^{n}
$$

\section{A new class of q-Fibonacci polynomials}

a) To each Morse code sequence of $n$ letters $c_{1} c_{2} \ldots c_{n}$ we associate the weight $q^{i_{1}+\cdots+i_{k}} s^{k} x^{n-k}$ if the $i_{j}$ are those indices for which $c_{j}=b$. This means that the weight $w_{i}$ at $i$ is $w_{i}(a)=$ $x, w_{i}(b)=q^{i} s$ and the total weight satisfies $w\left(c_{1} c_{2} \cdots c_{n}\right)=w_{1}\left(c_{1}\right) w_{2}\left(c_{2}\right) \cdots w_{n}\left(c_{n}\right)$. Then it is easy to see that $w\left(c_{1} c_{2} \cdots c_{n}\right)=\varphi\left(c_{1} c_{2} \cdots c_{n}\right) 1$.

If the word $c_{1} c_{2} \cdots c_{n}$ has $k$ elements $b$ then

$$
\varphi\left(c_{1} c_{2} \cdots c_{n}\right)=q^{i_{1}+\cdots i_{k}} s^{k} x^{n-k} \varepsilon^{n}
$$

In [6] we have defined polynomials $F_{n}(a, b)$ as the sum of all monomials $u \in M C$ of length $l(u)=n-1$. There we have called them abstract Fibonacci polynomials. As the referee pointed out, it would be better to call them noncommutative Fibonacci polynomials. By classifying with respect to the first or last letter respectively we see that $F_{n}(a, b)=a F_{n-1}(a, b)+b F_{n-2}(a, b)$ and also $F_{n}(a, b)=F_{n-1}(a, b) a+F_{n-2}(a, b) b$ with initial values $F_{0}(a, b)=0, F_{1}(a, b)=1$.

If we apply the homomorphism $\varphi$ we get $\varphi\left(F_{n}(a, b)\right) 1=F_{n}(x, s)$ with polynomials $F_{n}(x, s)$. These polynomials are the $q$-Fibonacci polynomials which we will study in this paper.

\section{Theorem 2.1}

The q-Fibonacci polynomials satisfy each of the recurrences

$$
\begin{gathered}
F_{n}(x, s)=x F_{n-1}(x, q s)+q s F_{n-2}(x, q s), \\
F_{n}(x, s)=x F_{n-1}(x, s)+q^{n-2} s F_{n-2}\left(x, \frac{s}{q}\right),
\end{gathered}
$$

and

$$
F_{n}(x, s)=x F_{n-1}(x, s)+q^{n-2} s x F_{n-3}(x, s)+q^{n-2} s^{2} F_{n-4}(x, s)
$$

and are given by the explicit formula

$$
F_{n}(x, s)=\sum_{k=0}^{\left\lfloor\frac{n-1}{2}\right\rfloor}\left[\begin{array}{c}
n-k-1 \\
k
\end{array}\right] q^{\left(\begin{array}{c}
k+1 \\
2
\end{array}\right) x^{n-1-2 k} s^{k}} .
$$

\section{Proof.}

The first equation follows by considering the first letter of the Fibonacci word. To prove (2.3) we get the first term if the last letter of the Fibonacci word is $a$. If the last letter is $b$ we need the following result of [6]: 
Lemma. The noncommutative Fibonacci polynomials are given by

$$
F_{n}(a, b)=\sum_{k=0}^{n-1} C_{k}^{n-k-1}(a, b)
$$

This implies $\varphi\left(F_{n}(a, b)\right)=\sum_{k=0}^{n-1} \varphi\left(C_{k}^{n-k-1}(a, b)\right)$.

If $u b$ is a word with $n-k-1$ letters (2.1) gives

$\varphi(u) s=q^{i_{1}+\cdots+i_{k}} s^{k} x^{n-2 k-3} \varepsilon^{n-k-3} s=q^{i_{1} \cdots+i_{k}}\left(\frac{s}{q}\right)^{k} x^{n-2 k-3} q^{n-3} s$.

Therefore

$\varphi\left(F_{n-2}(a, b) q s \varepsilon\right)=q^{n-2} s F_{n-2}\left(x, \frac{s}{q}\right)$ and $(2.3)$ follows.

Combining (2.2) and (2.3) we get recursion (2.4).

From (2.6) and (1.6) we get the explicit formula.

We can extend $F_{n}(x, s)$ to negative $n$ by assuming $(2.2)$ for all $n \in \mathbb{Z}$. This gives

$$
F_{-n}(x, s)=(-1)^{n-1} \frac{F_{n}(x, s)}{s^{n}} .
$$

In order to verify this we must show that

$\frac{F_{n}(x, s)}{s^{n}}=-x \frac{F_{n+1}(x, q s)}{(q s)^{n+1}}+q s \frac{F_{n+2}(x, q s)}{(q s)^{n+2}}$

or

$q^{n+1} s F_{n}(x, s)=-x F_{n+1}(x, q s)+F_{n+2}(x, q s)$,

which is $(2.3)$.

There is also another recursion of a different kind (cf.[7]). Let $D$ be the q-differentiation operator defined by $D f(x)=\frac{f(q x)-f(x)}{q x-x}$. Then we get

$$
F_{n}(x, s)=x F_{n-1}(x, s)+(q-1) s D F_{n-1}(x, s)+s F_{n-2}(x, s) .
$$

For the proof it suffices to compare coefficients. This leads to the identity

$q^{\left(\begin{array}{c}k+1 \\ 2\end{array}\right)}\left[\begin{array}{c}n-k \\ k\end{array}\right]=q^{\left(\begin{array}{c}k+1 \\ 2\end{array}\right)}\left[\begin{array}{c}n-k-1 \\ k\end{array}\right]+(q-1) q^{\left(\begin{array}{c}k \\ 2\end{array}\right)}\left[\begin{array}{l}n-k \\ k-1\end{array}\right][n-2 k+1]+q^{\left(\begin{array}{c}k \\ 2\end{array}\right)}\left[\begin{array}{c}n-k-1 \\ k-1\end{array}\right]$ which is easily verified. Here $[n]$ denotes $[n]=\frac{q^{n}-1}{q-1}$.

From this we may deduce a formula for the q-derivative:

$$
(q-1) D F_{n}(x, s)=q^{n-1} F_{n-1}\left(x, \frac{s}{q}\right)-F_{n-1}(x, s) .
$$

We have also a precise q-analogue of the Lucas polynomials (cf. [7]). It satisfies the same recurrence $L u c_{n}(x, s)=(x+(q-1) s D) L u c_{n-1}(x, s)+s L u c_{n-2}(x, s)$ but with initial values $L u c_{0}(x, 2)=$ $2, \operatorname{Luc}_{1}(x, 2)=x$ and is given by the explicit formula 


$$
\operatorname{Luc}_{n}(x, s)=\sum_{j=0}^{\left\lfloor\frac{n}{2}\right\rfloor} \frac{[n]}{[n-j]}\left[\begin{array}{c}
n-j \\
j
\end{array}\right] q^{\left(\begin{array}{c}
j \\
2
\end{array}\right)} x^{n-2 j} s^{j} .
$$

These polynomials are related to the q-Fibonacci polynomials by the formulas $L u c_{n}(x, s)=$ $F_{n+1}(x, s)+s F_{n-1}(x, s)$

and

$\operatorname{DLuc} c_{n}(x, s)=[n] F_{n}\left(x, \frac{s}{q}\right)$.

They also satisfy the rather ugly recursion

$$
\begin{aligned}
& L u c_{n+4}(x, s)=x L u c_{n+3}(x, s)-q^{n+1} \frac{[2]}{[n+1]} s L u c_{n+2}(x, s)+ \\
& +q^{n+1} \frac{[n+3]}{[n+1]} s x L u c_{n+1}(x, s)+q^{n+1} \frac{[n+3]}{[n+1]} s^{2} L u c_{n}(x, s) .
\end{aligned}
$$

b) For the usual q-Fibonacci numbers G. Andrews [2] has obtained a "bizarre" generalization of the well known formula for Fibonacci numbers $F_{n}=\frac{1}{2^{n-1}} \sum_{k=0}^{\left\lfloor\frac{n-1}{2}\right\rfloor}\left(\begin{array}{c}n \\ 2 k+1\end{array}\right) 5^{k}$. For our q-Fibonacci numbers $a$ somewhat less bizarre generalization exists.

Let $A$ be the operator $x+(q-1) s D$ on $\mathbb{C}[x, s]$. Since $A$ and the multiplication operator $s$ commute it is obvious that the same formulas hold as in the case $q=1$ of ordinary Fibonacci polynomials.

E.g. $F_{n}(x, s)=\sum_{k=0}^{\left\lfloor\frac{n-1}{2}\right\rfloor}\left(\begin{array}{c}n-k-1 \\ k\end{array}\right) s^{k} A^{n-2 k-1} 1$

or the Binet formula

$F_{n}(x, s)=\frac{1}{\sqrt{A^{2}+4 s}}\left(\left(\frac{A+\sqrt{A^{2}+4 s}}{2}\right)^{n}-\left(\frac{A-\sqrt{A^{2}+4 s}}{2}\right)^{n}\right) 1$

which is equivalent with

$F_{n}(x, s)=\frac{1}{2^{n-1}} \sum_{k=0}^{n}\left(\begin{array}{c}n \\ 2 k+1\end{array}\right) A^{n-2 k-1}\left(A^{2}+4 s\right)^{k} 1$.

Let $r(n, k, x, s)$ be the polynomial $r(n, k, x, s)=A^{n-2 k-1}\left(A^{2}+4 s\right)^{k} 1$.

It satisfies the recurrence

$r(n, k+1, x, s)=r(n, k, x, s)+4 s r(n-2, k, x, s)$, since

$A^{n-2(k+1)-1}\left(A^{2}+4 s\right)^{k+1}=A^{n-2 k-3}\left(A^{2}+4 s\right)\left(A^{2}+4 s\right)^{k}=$

$=A^{n-2 k-1}\left(A^{2}+4 s\right)^{k}+4 s A^{(n-2)-2 k-1}\left(A^{2}+4 s\right)^{k}$.

For the q-Fibonacci numbers $F_{n}(q)=\sum_{k=0}^{\left\lfloor\frac{n-1}{2}\right\rfloor}\left[\begin{array}{c}n-k-1 \\ k\end{array}\right] q^{\left(\begin{array}{c}k+1 \\ 2\end{array}\right)}$ this implies

$F_{n}(q)=\frac{1}{2^{n-1}} \sum_{k=0}^{\left\lfloor\frac{n-1}{2}\right\rfloor}\left(\begin{array}{c}n \\ 2 k+1\end{array}\right) r(n, k, 1,1)$, where $r(n, k, 1,1)$ is a polynomial in $q$ with integer coefficients. Of course for $q=1$ we get $r(n, k, 1,1)=5^{k}$, which does not depend on $n$. From (2.4) it is easy to derive that the degree $\operatorname{deg} F_{n}(q)$ as polynomial in $q$ is given by

$$
\operatorname{deg} F_{3 n}(q)=\frac{n(3 n-1)}{2}, \operatorname{deg} F_{3 n+1}(q)=\frac{n(3 n+1)}{2}, \operatorname{deg} F_{3 n+2}(q)=\frac{3 n(n+1)}{2} .
$$


c) The Fibonacci numbers satisfy $F_{2 n}=\sum_{k=0}^{n}\left(\begin{array}{l}n \\ k\end{array}\right) F_{n-k}$. This property has two nice generalizations

$$
F_{2 n}(x, s)=\sum_{k=0}^{n}\left[\begin{array}{l}
n \\
k
\end{array}\right] q^{\left(\begin{array}{c}
k+1 \\
2
\end{array}\right)} s^{k} x^{n-k} F_{n-k}\left(x, q^{n} s\right)
$$

and

$$
F_{2 n}(x, s)=\sum_{k=0}^{n}\left[\begin{array}{l}
n \\
k
\end{array}\right] q^{n k-\left(\begin{array}{c}
k+1 \\
2
\end{array}\right) s^{k} x^{n-k} F_{n-k}\left(x, \frac{s}{q^{k}}\right) .}
$$

The first one follows immediately from the formula

$$
F_{2 n}(a, b)=\sum_{k=0}^{n} C_{k}^{n}(a, b) F_{n-k}(a, b)
$$

for noncommutative Fibonacci polynomials proved in [8].

The second one follows from the companion formula

$$
F_{2 n}(a, b)=\sum_{k=0}^{n} F_{n-k}(a, b) C_{k}^{n}(a, b)
$$

For the proof observe that $\varphi\left(F_{n-k}(a, b)\right)=\sum_{l=0}^{n-k-1} \varphi\left(C_{k}^{n-k-l-1}(a, b)\right)$.

Each word $u \in \varphi\left(C_{l}^{n-k-l-1}(a, b)\right)$ has the form $q^{i_{1}+\cdots+i_{l}} s x^{l-k-2 l-1} \varepsilon^{n-k-l-1}$. Therefore $u s^{k} 1=q^{i_{1}+\cdots+i_{l}} s^{l} x^{n-k-2 l-1} q^{(n-k-l-1) k} s^{k}=q^{i_{1}+\cdots+i_{l}}\left(\frac{s}{q^{k}}\right)^{l} q^{n k-2\left(\begin{array}{c}k+1 \\ 2\end{array}\right)} s^{k} x^{n-2 l-k-1}$. This implies (2.10).

d) The formula $F_{n+k}=\sum_{j=0}^{k}\left(\begin{array}{l}k \\ j\end{array}\right) F_{n-j}$ can be generalized to

$$
F_{n}\left(x, \frac{s}{q^{k}}\right)=\sum_{j=0}^{k} x^{k-j}\left[\begin{array}{l}
k \\
j
\end{array}\right]\left(\frac{s}{q^{k}}\right)^{j} q^{\left(\begin{array}{c}
j+1 \\
2
\end{array}\right)} F_{n-k-j}(x, s) .
$$

Observe that some $F_{l}(x, s)$ will have negative subscripts $l$. This formula can be proved by induction starting from

$$
F_{n}\left(x, \frac{s}{q}\right)=x F_{n-1}(x, s)+s F_{n-2}(x, s) .
$$

For we have

$$
\begin{aligned}
& F_{n}\left(x, \frac{s}{q^{k+1}}\right)=\sum_{j=0}^{k} x^{k-j}\left[\begin{array}{c}
k \\
j
\end{array}\right]\left(\frac{s}{q^{k+1}}\right)^{j} q^{\left(\begin{array}{c}
j+1 \\
2
\end{array}\right)} F_{n-k-j}\left(x, \frac{s}{q}\right)= \\
& \sum_{j=0}^{k} x^{k-j}\left[\begin{array}{c}
k \\
j
\end{array}\right]\left(\frac{s}{q^{k+1}}\right)^{j} q^{\left(\begin{array}{c}
j+1 \\
2
\end{array}\right)}\left(x F_{n-k-j-1}(x, s)+s F_{n-k-j-2}(x, s)\right)=
\end{aligned}
$$




$$
\begin{aligned}
& \sum_{j=0}^{k} x^{k-j+1}\left[\begin{array}{c}
k \\
j
\end{array}\right]\left(\frac{s}{q^{k+1}}\right)^{j} q^{\left(\begin{array}{c}
j+1 \\
2
\end{array}\right)} F_{n-k-j-1}(x, s)+ \\
& +\sum_{j=1}^{k+1} x^{k-j+1}\left[\begin{array}{c}
k \\
j-1
\end{array}\right]\left(\frac{s}{q^{k+1}}\right)^{j} q^{\left(\begin{array}{c}
j+1 \\
2
\end{array}\right)+k-j+1} F_{n-k-j-1}(x, s)= \\
& =\sum_{j=0}^{k+1} x^{k-j+1}\left[\begin{array}{c}
k+1 \\
j
\end{array}\right]\left(\frac{s}{q^{k+1}}\right)^{j} q^{\left(\begin{array}{c}
j+1 \\
2
\end{array}\right)} F_{n-k-j-1}(x, s) .
\end{aligned}
$$

Together with (2.10) this implies

$$
\begin{aligned}
& F_{2 n}(x, s)=\sum_{k}\left[\begin{array}{c}
n \\
k
\end{array}\right] q^{n k-\left(\begin{array}{c}
k+1 \\
2
\end{array}\right)} s^{k} x^{n-k} \sum_{j=0}^{k} x^{k-j}\left[\begin{array}{c}
k \\
j
\end{array}\right]\left(\frac{s}{q^{k}}\right)^{j} q^{\left(\begin{array}{c}
j+1 \\
2
\end{array}\right)} F_{n-2 k-j}(x, s)= \\
& =\sum_{k, j}\left[\begin{array}{l}
n \\
k
\end{array}\right]\left[\begin{array}{c}
k \\
j
\end{array}\right] q^{n k-j k-\left(\begin{array}{c}
k+1 \\
2
\end{array}\right)+\left(\begin{array}{c}
j+1 \\
2
\end{array}\right)} s^{k+j} x^{n-j} F_{n-2 k-j}(x, s) .
\end{aligned}
$$

If in (2.11) we replace $n$ by $2 n, s$ by $q^{n} s$ and $k$ by $n$ we get again (2.9). In the other direction we have

$$
F_{n}(x, q s)=\sum_{j=0}^{n-1}(-1)^{j}(q s)^{j} x^{-1-j} F_{n+1-j}(x, s) .
$$

This follows from the recursion (2.2) by induction. It is true for $n=1$ because $x F_{1}(x, q s)=F_{2}(x, s)$. If it is true for $n-1$ we get

$x \sum_{j=0}^{n-1}(-1)^{j}(q s)^{j} x^{-1-j} F_{n+1-j}(x, s)=$

$=F_{n+1}(x, s)-q s \sum_{j=0}^{n-2}(-1)^{j}(q s)^{j} x^{-1-j} F_{n-j}(x, s)=$

$=F_{n+1}(x, s)-q s F_{n-1}(x, q s)=x F_{n}(x, q s)$.

e) Another interesting formula is

$$
x^{k} F_{n}\left(x, q^{k} s\right)=\sum_{j=0}^{k}(-1)^{j}\left[\begin{array}{l}
k \\
j
\end{array}\right] q^{j^{2}} s^{j} F_{n+k-2 j}\left(x, q^{j} s\right) .
$$

We prove it by induction. The formula holds for $k=1$ and all $n$. If it is true for $k$ then we get

$$
\begin{aligned}
& x^{k+1} F_{n}\left(x, q^{k+1} s\right)=\sum_{j=0}^{k}(-1)^{j}\left[\begin{array}{c}
k \\
j
\end{array}\right] q^{j^{2}}(q s)^{j} x F_{n+k-2 j}\left(x, q^{j+1} s\right)= \\
& =\sum_{j=0}^{k}(-1)^{j}\left[\begin{array}{c}
k \\
j
\end{array}\right] q^{j^{2}}(q s)^{j}\left(F_{n+k+1-2 j}\left(x, q^{j} s\right)-q^{j+1} s F_{n+k-1-2 j}\left(x, q^{j+1} s\right)\right)= \\
& =\sum_{j \geq 0}(-1)^{j}\left(\left[\begin{array}{c}
k \\
j
\end{array}\right] q^{j}+\left[\begin{array}{c}
k \\
j-1
\end{array}\right]\right) q^{j^{2}} s^{j} F_{n+k+1-2 j}\left(x, q^{j} s\right)= \\
& =\sum_{j \geq 0}(-1)^{j}\left[\begin{array}{c}
k+1 \\
j
\end{array}\right] q^{j^{2}} s^{j} F_{n+k+1-2 j}\left(x, q^{j} s\right) .
\end{aligned}
$$

For $n=1$ we get $x^{k}=\sum_{j=0}^{k}(-1)^{j}\left[\begin{array}{l}k \\ j\end{array}\right] q^{j^{2}} s^{j} F_{k+1-2 j}\left(x, q^{j} s\right)$. 
This may be rewritten in the form

$$
\begin{aligned}
& x^{k}=\sum_{j=0}^{\left\lfloor\frac{k+1}{2}\right\rfloor}(-1)^{j}\left[\begin{array}{l}
k \\
j
\end{array}\right] q^{j^{2}} s^{j} F_{k+1-2 j}\left(x, q^{j} s\right)+ \\
& +\sum_{j=0}^{\left\lfloor\frac{k-1}{2}\right\rfloor}(-1)^{j}\left[\begin{array}{l}
k \\
j
\end{array}\right] q^{k+k j-j^{2}-j} s^{j+1} F_{k-2 j-1}\left(x, q^{k-j} s\right) .
\end{aligned}
$$

For $n=0$ this reduces to

$$
\sum_{j=0}^{\left\lfloor\frac{k-1}{2}\right\rfloor}(-1)^{j}\left[\begin{array}{c}
k \\
j
\end{array}\right] s^{j}\left(q^{j^{2}} F_{k-2 j}\left(x, q^{j} s\right)-q^{j k-j^{2}} F_{k-2 j}\left(x, q^{k-j} s\right)\right)=0 .
$$

For $q \neq 1$ this is nontrivial identity.

f) Define $b_{n, k}$ by $b_{0, k}=[k=0], b_{1, k}=[k=0]$, the recursion $b_{n+1, k}=q^{k} b_{n, k}+b_{n, k-1}$ for $k \leq\left\lfloor\frac{n}{2}\right\rfloor$, and $b_{2 n+1, n+1}=0$.

Then we get

$$
x^{n}=\sum_{2 k \leq n}(-1)^{k}(q s)^{k} q^{2\left(\begin{array}{c}
k \\
2
\end{array}\right)} b_{n, k} F_{n+1-2 k}\left(q^{k} s\right) .
$$

Obviously (2.14) holds for $n=1$. So we may assume that it holds for $n$. Since $b_{n, k}$ does not depend on $s$ the same formula holds for $q s$ in place of $s$. From (2.2) we therefore get

$$
\begin{aligned}
& x \sum_{2 k \leq n}(-1)^{k}\left(q^{2} s\right)^{k} q^{2\left(\begin{array}{c}
k \\
2
\end{array}\right)} b_{n, k} F_{n+1-2 k}\left(q^{k} q s\right)= \\
& =\sum_{k=0}^{\left\lfloor\frac{n}{2}\right\rfloor}(-1)^{k}\left(q^{2} s\right)^{k} q^{2\left(\begin{array}{c}
k \\
2
\end{array}\right)} b_{n, k} F_{n+2-2 k}\left(q^{k} s\right)- \\
& -\sum_{k=0}^{\left\lfloor\frac{n}{2}\right\rfloor}(-1)^{k}\left(q^{2} s\right)^{k} q^{2\left(\begin{array}{c}
k \\
2
\end{array}\right)} b_{n, k} q^{k+1} s F_{n-2 k}\left(q^{k+1} s\right)= \\
& =\sum_{k=0}^{\left\lfloor\frac{n}{2}\right\rfloor}(-1)^{k}\left(q^{2} s\right)^{k} q^{2\left(\begin{array}{c}
k \\
2
\end{array}\right)} b_{n, k} F_{n+2-2 k}\left(q^{k} s\right)+ \\
& +\sum_{k=1}^{\left\lfloor\frac{n}{2}\right\rfloor+1}(-1)^{k}(q s)^{k} q^{2\left(\begin{array}{c}
k \\
2
\end{array}\right)} b_{n, k} F_{n-2 k+2}\left(q^{k} s\right)= \\
& =\sum_{2 k \leq n+1}(-1)^{k}(q s)^{k} q^{2}\left(\begin{array}{l}
k \\
2
\end{array}\right) b_{n+1, k} F_{n+2-2 k}\left(q^{k} s\right) .
\end{aligned}
$$

There is no explicit formula for $b_{n, k}$. By comparing coefficients we get from $(2.14)$ the following characterization

$$
\sum_{j=0}^{k}(-1)^{j}\left[\begin{array}{c}
n-k-j \\
k-j
\end{array}\right] q^{\left(\begin{array}{c}
j \\
2
\end{array}\right)} b_{n, j}=[k=0] .
$$

Let now $a_{n, n-2 k}=b_{n, k}$ or $a_{n, k}=b_{n, \frac{n-k}{2}}$. Then we get $a_{n, k}=q^{\frac{n-k}{2}} a_{n-1, k-1}+a_{n-1, k+1}$ with $a_{n,-1}=0$ for all $n$ and initial values $a_{0, k}=[k=0]$ and $a_{1, k}=[k=1]$. For $q=1$ it is well known that $a_{2 n, 0}=\frac{1}{n+1}\left(\begin{array}{c}2 n \\ n\end{array}\right)$ is a Catalan number. Therefore $a_{2 n, 0}$ is a q-analogue of the Catalan numbers (cf. e.g. [6]). 


\section{Special values}

It turns out that for $x=1, s=-1$ or $x=1, s=\frac{-1}{q}$ we get very simple results.

Theorem 3.1 Let $r(k)=\frac{k(3 k-1)}{2}$ denote a pentagonal number. Then

$$
F_{3 n}(1,-1)=\sum_{k=-n+1}^{n}(-1)^{k} q^{r(k)}, F_{3 n+1}(1,-1)=F_{3 n+2}(1,-1)=\sum_{k=-n}^{n}(-1)^{k} q^{r(k)}
$$

Remark. This is a curious result. It means that the values $F_{n}(1,-1)$ are just the partial sums of Euler's pentagonal number series

$\prod_{n \geq 1}\left(1-q^{n}\right)=1-q-q^{2}+q^{5}+q^{7}--++\ldots=$

$=\sum_{k=-\infty}^{\infty}(-1)^{k} q^{\frac{k(3 k-1)}{2}}=\sum_{k \in \mathbb{Z}}(-1)^{k} q^{r(k)}$.

Theorem 3.2 For $x=1, s=\frac{-1}{q}$ we get

$$
F_{3 n}\left(1,-\frac{1}{q}\right)=0, F_{3 n+1}\left(1,-\frac{1}{q}\right)=(-1)^{n} q^{r(n)}, F_{3 n+2}\left(1,-\frac{1}{q}\right)=(-1)^{n} q^{r(-n)} .
$$

Remark. Formula (3.2) has been proved by Shalosh B. Ekhad and D. Zeilberger [14] with a computer proof and by S. O. Warnaar [13] as a special case of a cubic summation formula in the form

$\sum_{k=0}^{\left\lfloor\frac{n}{2}\right\rfloor}(-1)^{k} q^{\left(\begin{array}{c}k \\ 2\end{array}\right)}\left[\begin{array}{c}n-k \\ k\end{array}\right]=\left\{\begin{array}{cl}(-1)^{\left\lfloor\frac{n}{3}\right\rfloor} q^{\frac{n(n-1)}{6}}, n \neq \equiv 2 \quad(\bmod 3) \\ 0, n \equiv 2 \quad(\bmod 3) .\end{array}\right.$

I learned from C. Krattenthaler that the cubic summation formula referred to by S. O. Warnaar is formula (5.22) of [10], and that from the hypergeometric view this seems to be the most natural approach to (3.2). Since I am not familiar with bibasic series I want to give some elementary proofs which do not need much theory. From (2.11) and (2.12) we see that Theorem 3.1 and Theorem 3.2 are equivalent. So it suffices to prove one of them.

a) We first give a direct proof of (3.2). For $x=1, s=-\frac{1}{q}$ formula (2.4 ) reduces to $F_{n}\left(1,-\frac{1}{q}\right)=F_{n-1}\left(1,-\frac{1}{q}\right)-q^{n-3} F_{n-3}\left(1,-\frac{1}{q}\right)+q^{n-4} F_{n-4}\left(1,-\frac{1}{q}\right)$.

From this we get

$$
F_{n+3}\left(1,-\frac{1}{q}\right)=\left(1-q^{n}\right) F_{n}\left(1,-\frac{1}{q}\right)+q^{n-3} F_{n-3}\left(1,-\frac{1}{q}\right) .
$$

Since $F_{4}\left(1,-\frac{1}{q}\right)=-q F_{1}\left(1,-\frac{1}{q}\right), F_{5}\left(1,-\frac{1}{q}\right)=-q^{2} F_{2}\left(1,-\frac{1}{q}\right)$ we even get

$$
F_{n+3}\left(1,-\frac{1}{q}\right)=-q^{n} F_{n}\left(1,-\frac{1}{q}\right) .
$$


This implies immediately

$F_{3 n}\left(1,-\frac{1}{q}\right)=0$,

$F_{3 n+1}\left(1,-\frac{1}{q}\right)=(-1)^{n} q^{\sum_{i=1}^{n} 3 i-2}=(-1)^{n} q^{r(n)}$,

$F_{3 n+2}\left(1,-\frac{1}{q}\right)=(-1)^{n} q^{\sum_{i=1}^{n} 3 i-1}=(-1)^{n} q^{r(-n)}$.

b) But there is a more illuminating combinatorial proof of (3.1), which is an adaptation of Franklin's proof of the pentagonal number theorem to this case (cf. [1] or [4]).

Let $u$ be a word of length $n-1, u \neq a^{n-1}$. Then we can write it in the form $u=a^{i-1} z b^{l} a^{j}$ where $i, l \geq 1$ are chosen to be maximal. In case $i \leq l$ we call the first $b$ from the left $b_{1}$ and the $i$-th $b$ from the right $b_{2}$. If $b_{1} \neq b_{2}$ we call $u$ good of type $b$ and define $\psi(u)$ by changing $b_{1}$ to $a$ and $b_{2}$ to $a b$. Then $\psi(u)$ has the same length and the same weight as $u$ but the number of $b$ 's is one less. If $b_{1}=b_{2}$ we call $u$ bad of type $b$. It is easy to see that $u$ is bad of type $b$ if and only if $u=a^{i-1} b^{i} a^{j}, i \geq 1$.

Then $\psi(u)$ has the form $u=a^{i-1} z b^{l} a^{j}$ with $i>l \geq 1$. If $u$ has this form let $a_{1}$ be the $l$-th element $a$ from the left and $a_{2}$ the $a$ immediately in front of $b^{l}$. If $a_{1} \neq a_{2}$ we call $u$ good of type $a$ and define $\psi(u)$ as follows. We change $a_{1}$ to $b$ and drop $a_{2}$. Then it is clear that $\psi$ maps the set of good words of type $a$ bijectively onto the set of good words of type $b$ in such a way that words with an even number of $b^{\prime}$ s are mapped onto words with an odd number of $b^{\prime}$ s and vice versa. If $a_{1}=a_{2}$ we call $u$ bad of type $a$. Then $u$ is bad of type $a$ if and ony if $u=a^{l} b^{l} a^{j}$ for some $l \geq 1$.

Let for example $n=10$ and $u=a b a b b a$, then $u=a b a b b a=a b_{1} a b_{2} b a$ and therefore $\psi(u)=a \underline{a} a \underline{a b} b a$. The weight of these words is 11. If $u=a b a b a a$ then $u$ is good of type $a$ with length 8 and weight $6, u=a_{1} b a_{2} b a$. Here we get $\psi(u)=\psi\left(a_{1} b a_{2} b a\right)=\underline{b} b \underline{b} a$.

The weights of the bad words are $w\left(a^{i-1} b^{i} a^{j}\right)=\frac{i(3 i-1)}{2}$ and $w\left(a^{l} b^{l} a^{j}\right)=\frac{l(3 l+1)}{2}$.

For a Morse code sequence of length $3 n-1$ the bad word with maximal weight is $a^{n-1} b^{n}$, for the length $3 n$ and $3 n+1$ the corresponding words are $a^{n} b^{n}$ and $a^{n} b^{n} a$. The weights of these words are $r(n)$ and $r(-n)$ respectively.

Therefore we get the desired result

$F_{3 n}(1,-1)=\sum_{k=-n+1}^{n}(-1)^{k} q^{r(k)}, F_{3 n+1}(1,-1)=F_{3 n+2}(1,-1)=\sum_{k=-n}^{n}(-1)^{k} q^{r(k)}$.

c) Another proof of (3.2) has been communicated to me independently both by $\mathrm{R}$. Chapman and C. Krattenthaler, which I reproduce in my notation.

From $(1+s)(1+q s) \cdots\left(1+q^{n-1} s\right)=\sum_{k=0}^{n}\left[\begin{array}{l}n \\ k\end{array}\right] q^{\left(\begin{array}{c}k \\ 2\end{array}\right)} s^{k}(\mathrm{cf} .(1.5))$ follows $\left[\begin{array}{c}n-k-1 \\ k\end{array}\right] q^{\left(\begin{array}{c}k \\ 2\end{array}\right)}=\sum_{0 \leq i_{1}<i_{2}<\cdots<i_{k}<n-k-1} q^{i_{1}+\cdots+i_{k}}$.

In the sum $\sum(-1)^{k}\left[\begin{array}{c}n-k-1 \\ k\end{array}\right] q^{\left(\begin{array}{c}k \\ 2\end{array}\right)}$ all terms $q^{i_{1}+\cdots i_{k}}$ with $i_{1}=0$ cancel, because $(-1)^{k} q^{i_{1}+\cdots i_{k}}+$ $(-1)^{k-1} q^{i_{2}+\cdots i_{k}}=0$. The only terms remaining are those with $i_{1}>0$ and $i_{k}+k=n-2$. 
The above proof or the original proof of Franklin lets $i_{k}+k$ invariant and gives immediately

$F_{3 n}\left(1,-\frac{1}{q}\right)=0, F_{3 n+1}\left(1,-\frac{1}{q}\right)=w\left(a^{n-1} b^{n}\right)=(-1)^{n} q^{r(n)}$,

$F_{3 n+2}\left(1,-\frac{1}{q}\right)=w\left(a^{n-1} b^{n} a\right)=(-1)^{n} q^{r(-n)}$.

\section{A generating function}

Now we want to calculate the generating function

$F(x, s, z)=\sum_{n \geq 0} F_{n+1}(x, s) z^{n}$ of the q-Fibonacci polynomials. We have

$F(x, s, z)=\sum_{n \geq 0}^{n \geq 0} F_{n+1}(x, s) z^{n}=1+\sum_{n \geq 1} x F_{n}(x, q s) z^{n}+\sum_{n \geq 1} q s F_{n-1}(x, q s) z^{n}=$

$=1+x z F(x, q s, z)+q s z^{2} F(x, q s, z)=1+x z \varepsilon F(x, s, z)+q s z^{2} \varepsilon F(x, s, z)$

i.e.

$$
F(x, s, z)=1+\left(x z+q s z^{2}\right) F(x, q s, z)
$$

or

$\left(1-x z \varepsilon-q z^{2} s \varepsilon\right) F(x, s, z)=1$

and therefore

$$
F(x, s, z)=\sum_{n \geq 0}\left(x z \varepsilon+z^{2} s \varepsilon\right)^{n} 1 .
$$

Now we have $z \varepsilon \cdot s z^{2} \varepsilon=q s z^{2} \varepsilon \cdot z \varepsilon$. Therefore the q-binomial theorem gives $\left(x z \varepsilon+z^{2} q s \varepsilon\right)^{n} 1=\sum_{k=0}^{n}\left[\begin{array}{l}n \\ k\end{array}\right]\left(q z^{2} s \varepsilon\right)^{k}(x z)^{n-k} 1=\sum_{k=0}^{n}\left[\begin{array}{l}n \\ k\end{array}\right] q^{\left(\begin{array}{c}k+1 \\ 2\end{array}\right)} s^{k} x^{n-k} z^{n+k}$.

Using the well known formula

$\frac{1}{(1-z)(1-q z) \cdots\left(1-q^{k} z\right)}=\sum\left[\begin{array}{c}n+k \\ k\end{array}\right] z^{n}$ this implies

$F(x, s, z)=\sum_{n} \sum_{k=0}^{n}\left[\begin{array}{l}n \\ k\end{array}\right] x^{n-k} q^{\left(\begin{array}{c}k+1 \\ 2\end{array}\right)} z^{n+k} s^{k}=$

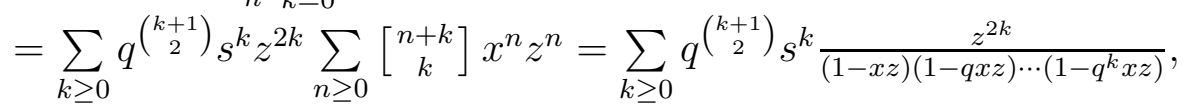

i.e.

$$
F(x, s, z)=\sum_{n \geq 0} F_{n+1}(x, s) z^{n}=\sum_{k \geq 0} q^{\left(\begin{array}{c}
k+1 \\
2
\end{array}\right)} s^{k} \frac{z^{2 k}}{(1-x z)(1-q x z) \cdots\left(1-q^{k} x z\right)} .
$$

On the other hand we have

$$
\left(x z \varepsilon+q z^{2} s \varepsilon\right)^{n} 1=z^{n}(x+q s z)\left(x+q^{2} s z\right) \cdots\left(x+q^{n} s z\right) .
$$

From (4.3) and (4.2) we get

$$
\sum_{k \geq 0} q^{\left(\begin{array}{c}
k+1 \\
2
\end{array}\right)} s^{k} \frac{z^{2 k}}{(1-x z)(1-q x z) \cdots\left(1-q^{k} x z\right)}=\sum_{n \geq 0} z^{n}(x+q s z)\left(x+q^{2} s z\right) \cdots\left(x+q^{n} s z\right)
$$


If we set $x=1, a=-q s z$ this reduces to

$$
\sum_{k \geq 0}(-1)^{k} q^{\left(\begin{array}{c}
k \\
2
\end{array}\right)} \frac{(a z)^{k}}{(1-z)(1-q z) \cdot\left(1-q^{k} z\right)}=\sum_{k \geq 0} z^{n}(1-a)(1-q a) \cdots\left(1-q^{n-1} a\right) .
$$

From (4.6) and (4.3) we also get

$\sum_{k \geq 0} z^{k}(1-z)(1-q z) \cdots\left(1-q^{k-1} z\right)=\sum_{k \geq 0} F_{k+1}\left(1,-\frac{1}{q}\right) z^{k}$.

Letting $a=z$ this gives

$$
\sum_{k \geq 0} z^{k}(1-z)(1-q z) \cdots\left(1-q^{k-1} z\right)=\sum_{k \geq 0}(-1)^{k}\left(q^{r(k)} z^{3 k}+q^{r(-k)} z^{3 k+1}\right) .
$$

Remark. This identity which is equivalent with (3.2) is well known (c.f. [1], p. 29, Example 10, [14], p. 951 or [4]).

In our notation the usual proof may be formulated in a very convincing way:

The recursion (2.2) implies identity (4.1) for the generating function. In the same way the other recursion (2.3) gives

$$
(1-x z) F(x, s, z)=1+q s z^{2} F\left(x, \frac{s}{q}, q z\right) .
$$

From (4.8) for $x=1, s=-1$ we get

$(1-z) F(1,-1, z)=1-q z^{2} F\left(1,-\frac{1}{q}, q z\right)$

and from (4.1) for $x=1, s=-\frac{1}{q}$ we get in the same way

$F\left(1,-\frac{1}{q}, z\right)=1+z(1-z) F(1,-1, z)$.

This implies

$F\left(1,-\frac{1}{q}, z\right)=1+z-q z^{3} F\left(1,-\frac{1}{q}, q z\right)$,

which is equivalent with

$$
F_{n+3}\left(1, \frac{-1}{q}\right)=-q^{n} F_{n}\left(1, \frac{-1}{q}\right)
$$

and therefore with (3.2).

Formulae (3.2) and (3.1) are generalizations of the trivial fact that the recursion $f_{n}=f_{n-1}-f_{n-2}, f_{0}=0, f_{1}=1$ has the periodic solution $0,1,1,0,-1,-1,0,1,1,0, \cdots$ with period 6 .

For $q=1$ formulas (4.6) and (4.7) reduce to

$\frac{1}{1-z+z^{2}}=\sum(-1)^{k} \frac{z^{2 k}}{(1-z)^{k+1}}=\sum z^{k}(1-z)^{k}=\frac{1+z}{1+z^{3}}=$ $=1+z-z^{3}-z^{4}+z^{6}+z^{7}--++\cdots$ 


\section{A Hankel determinant}

\section{Theorem 5.1}

Let $\beta(n, k)=\frac{n(n+k-2)(n+k-3)}{6}$ and $\alpha(n, k)=\beta(n, k)$ if $(n-2 k) \not \equiv 1 \quad(\bmod 3), \alpha(n, k)=0$ if $n-2 k \equiv 1 \quad(\bmod 6)$ and $\alpha(n, k)=\beta(n, k)+\frac{n}{6}$ if $n-2 k \equiv 4 \quad(\bmod 6)$.

Then the Hankel determinant $d_{n, k}=\operatorname{det}\left(F_{i+j+k}(1,-1 / q)\right)_{i, j=0}^{n-1}$ satisfies

$$
d_{n, k}=(-1)^{k} \varepsilon(n-2 k) q^{\alpha(n, k)} \prod_{i=1}^{\left\lfloor\frac{n}{2}\right\rfloor}\left(q^{i}-1\right)^{n-2 i}
$$

where the sign $\varepsilon(n)$ is given by $\varepsilon(2 n)=(-1)^{n}, \varepsilon(6 n+1)=0, \varepsilon(6 n+3)=1, \varepsilon(6 n+5)=-1$.

Proof. We use the condensation method as outlined in Krattenthaler [11] or Zeilberger [15]. In a similar context this method has been used in [12], which inspired our proof. Equation [11] (2.16) gives in this case equation [12] (2.3), which states that

$$
d_{n, k+1} d_{n . k-1}-d_{n-1, k+1} d_{n+1, k-1}=\left(d_{n, k}\right)^{2}
$$

holds for all $n, k$.

This identity lends itself to prove (5.1) by induction with respect to $n$ for all $k$.

From $F_{n+3}\left(1,-\frac{1}{q}\right)=-q^{n} F_{n}\left(1,-\frac{1}{q}\right)$ it is trivial that

$d_{n, k+3}=(-1)^{n} q^{2\left(\begin{array}{c}n \\ 2\end{array}\right)+k n} d_{n, k}$. So it would suffice to prove (5.2) only for $k=0,1,2$. But the general case is not harder to prove.

From $F_{3 n}\left(1,-\frac{1}{q}\right)=0$ and

$F_{n}\left(1,-\frac{1}{q}\right)=(-1)^{\left\lfloor\frac{n-1}{3}\right\rfloor} q^{\frac{(n-1)(n-2)}{6}}$ for $n \not \equiv 0 \quad(\bmod 3)$ we conclude that

$d_{1, k}=F_{k}\left(1,-\frac{1}{q}\right)=(-1)^{k} \varepsilon(1-2 k) q^{\alpha(1, k)}$.

In the same way we see by direct calculation that

$d_{2, k}=(-1)^{k} \varepsilon(2-2 k) q^{\alpha(2, k)}$.

Now from (5.2) we immediately see that $d_{n, k}$ for all $n \geq 3$ and all $k$ is uniquely determined. Thus all that remains is to verify formula (5.2) if we set

$d_{n, k}=(-1)^{k} \varepsilon(n-2 k) q^{\alpha(n, k)} \prod_{i=1}^{\left\lfloor\frac{n}{2}\right\rfloor}\left(q^{i}-1\right)^{n-2 i}$.

This reduces to

$$
\begin{aligned}
& \varepsilon(n-2 k-2) \varepsilon(n-2 k+2) q^{\alpha(n, k+1)+\alpha(n, k-1)}= \\
& \quad=\varepsilon(n-2 k-3) \varepsilon(n-2 k+3) q^{\alpha(n-1, k+1)+\alpha(n+1, k-1)}+q^{2 \alpha(n, k)} \varepsilon(n-2 k)^{2}
\end{aligned}
$$

for $n \equiv 1 \quad(\bmod 2)$

and to 


$$
\begin{aligned}
& \varepsilon(n-2 k-2) \varepsilon(n-2 k+2) q^{\alpha(n, k+1)+\alpha(n, k-1)}= \\
& \quad=\varepsilon(n-2 k-3) \varepsilon(n-2 k+3) q^{\alpha(n-1, k+1)+\alpha(n+1, k-1)}\left(q^{\frac{n}{2}}-1\right)+q^{2 \alpha(n, k)} \varepsilon(n-2 k)^{2}
\end{aligned}
$$

for $n \equiv 0 \quad(\bmod 2)$.

We consider several cases, where the resulting identity can easily be verified:

1) $n \equiv 2 k+1 \quad(\bmod 6)$ :

This implies $d_{n, k}=0$ and

$(n-1)-2(k+1) \equiv(n+1)-2(k-1) \equiv 4 \quad(\bmod 6)$.

Thus (5.3) reduces to

$-q^{\beta(n, k+1)+\beta(n, k-1)}+q^{\beta(n-1, k+1)+\beta(n+1, k-1)+\frac{n-1}{6}+\frac{n+1}{6}}=0$.

$2) n \equiv 2 k+3 \quad(\bmod 6):$

Here (5.3) reduces to

$q^{\beta(n-1, k+1)+\beta(n+1, k-1)}=q^{2 \beta(n, k)}$.

3) The same is true for $n \equiv 2 k+5 \quad(\bmod 6)$.

4) $n \equiv 2 k+4 \quad(\bmod 6):$

Here (5.4) reduces to $q^{\beta(n, k+1)+\beta(n, k-1)}=q^{2 \beta(n, k)+\frac{n}{3}}$.

$5)$ For $n \equiv 2 k \quad(\bmod 6)$ we have $n-2(k+1) \equiv 4 \quad(\bmod 6)$ and $(5.4)$ reduces to $q^{\left(\beta(n, k+1)+\frac{n}{6}\right)+\beta(n, k-1)}-\left(q^{\frac{n}{2}}-1\right) q^{(\beta(n+1, k-1)+\beta(n-1, k+1)}=q^{2 \beta(n, k)}$.

6) For $n \equiv 2 k+2 \quad(\bmod 6)$ we have $n-2(k-1) \equiv 4 \quad(\bmod 6)$ and $(5.4)$ reduces to $q^{\beta(n, k+1)+\left(\beta(n, k-1)+\frac{n}{6}\right)}-\left(q^{\frac{n}{2}}-1\right) q^{\beta(n+1, k-1)+\beta(n-1, k+1)}=q^{2 \beta(n, k)}$.

\section{References}

[1] G. E. Andrews, The Theory of Partitions, Addison-Wesley 1976

[2] G. E. Andrews, Fibonacci numbers and Rogers-Ramanujan identities, To appear in Fibonacci Quaterly

[3] L. Carlitz, Fibonacci notes 4, Fibonacci Quaterly 12(1975), 97 - 102

[4] R. Chapman, Franklin's argument proves an identity of Zagier, Electron J. Comb. $7(2000)$, R 54

[5] J. Cigler, Operatormethoden für q-Identitäten, Mh. Math. 88 (1979), 87-105

[6] J. Cigler, q-Fibonacci polynomials, Fibonacci Quarterly 41(2003), 31 - 40 
[7] J. Cigler, Einige q-Analoga der Lucas- und Fibonacci-Polynome, to appear in Sitzungsberichte Österr. Akad. Wiss.

[8] J. Cigler, Some algebraic aspects of Morse code sequences, to appear in DMTCS 2003

[9] Shalosh B. Ekhad, D. Zeilberger, The number of solutions of $X^{2}=0$ in triangular matrices over GF(q), Electron. J. Comb. 3(1996), R2

[10] G. Gasper, Summation, transformation, and expansion formulas for bibasic series, Trans. Amer. Math. Soc. 312(1989), 257 - 277

[11] C. Krattenthaler, Advanced determinant calculus, Seminaire Lotharingien de Combinatoire B42q, (1999)

[12] U. Tamm, Some aspects of Hankel matrices in coding theory and combinatorics, Electron. J. Comb. 8(2001), A1

[13] S. O. Warnaar, q-hypergeometric proofs of polynomial analogues of the triple product identity, Lebesgue's identity and Euler's pentagonal number theorem, arXiv:math. CO/0203229, 2002

[14] D. Zagier, Vassiliev invariants and a strange identity related to the Dedekind eta-function, Topology 40(2001), 945 - 960

[15] D. Zeilberger, Dodgsons determinant-evaluation rule proved by TWO-TIMING MEN and WOMEN, Electron. J. Comb. 4(2), 1997, R22 